\title{
Avaliação de Usabilidade do app ParecerEdu
}

\author{
Marina Braun Otokovieski, Amanda Meincke Melo, Ícaro Machado Crespo
}

\author{
${ }^{1}$ Universidade Federal do Pampa (UNIPAMPA) - Campus Alegrete \\ Av. Tiarajú, 810, Ibirapuitã - Alegrete, RS - Brasil \\ \{icarocrespo, marinaotok\}@gmail.com, amandamelo@unipampa.edu.br
}

\begin{abstract}
The software development advances more and more, occupying space in several domains. In the Education field, educational and management software are facilitators for classroom activities. Thus, the application ParecerEdu aims to help educators in managing opinions on the individual performance of students in the classroom. In order to inspect it to contribute to its enhancement, an Usability Heuristic Evaluation was conducted among the System Usability Scale application. The results obtained demonstrate the need for refactorings and are explored in this work.
\end{abstract}

Resumo. O desenvolvimento de software avança cada vez mais, ocupando espaço em diversos domínios. No âmbito da Educação, software educacionais e de gestão se mostram facilitadores para as atividades em sala de aula. Sendo assim, o aplicativo ParecerEdu visa auxiliar educadores na gestão de pareceres sobre o desempenho individual dos alunos em sala de aula. Com o intuito de inspecioná-lo para contribuir ao seu aprimoramento, foi conduzida uma Avaliação Heurística de Usabilidade em conjunto com a aplicação da escala System Usability Scale. Os resultados obtidos, demonstram necessidade de refatorações na interface, para melhor atender o usuário. Neste trabalho demonstramos como as metodologias foram aplicadas e quais podem ser os trabalhos futuros.

\section{Introdução}

Com o avanço das tecnologias digitais e seu uso frequente em diversos âmbitos, tornou-se corriqueira a adoção de ferramentas eletrônicas que simplifiquem as atividades cotidianas dos profissionais da Educação. Hoje em dia, é essencial que o professor se aproprie da presença das tecnologias no ambiente escolar, tornando esse um fator benéfico ao seu trabalho [Sousa 2011]. Além disso, sabe-se que software de gestão auxiliam na tomada de decisões, devido ao recolhimento de dados, proporcionando aumento de produtividade nas atividades [Nagar et al. 2018].

Tendo isso em vista, [Oliveira and Melo 2020] apresentaram o desenvolvimento de um aplicativo mobile denominado ParecerEdu. Este tem como propósito auxiliar professores das séries iniciais do ensino fundamental na geração de pareceres a partir de dados de acompanhamento de estudantes em sala de aula. Com a geração de pareceres se torna viável analisar critérios de aprendizagem e comportamento dos alunos em sala de aula, organizando o dia a dia da turma. Todos esses critérios podem ser cadastrados de acordo com a necessidade de cada professor, dentro do escopo da disciplina. Um professor de matemática, por exemplo, poderia cadastrar critérios como "conhecimento de tabuada" e "cálculos de soma". A proposta partiu do levantamento de um problema focal, denominado “Tecnologias Digitais para Gestão Escolar", onde foram identificadas as 
dificuldades enfrentadas no ensino fundamental de duas escolas da educação básica, no município de Alegrete/RS.

Para avaliar a ferramenta desenvolvida, foram realizadas sessões de Avaliação Cooperativa [Muller et al. 1997], que contaram com a participação de duas professoras, atuantes nas escolas envolvidas. Uma das professoras, participou diretamente da elaboração da interface do projeto. Constatou-se, desse modo, a necessidade de melhorias nas operações realizadas no aplicativo, além de melhorias estéticas, como o uso das cores utilizadas nos gráficos [Oliveira and Melo 2020]. Para que seja possível dar segmento ao projeto e inseri-lo no meio educacional, portanto, novas avaliações são necessárias. Essas avaliações permitirão identificar problemas e lacunas no funcionamento do aplicativo, assim como orientar correções e a evolução do aplicativo.

Sendo assim, o presente artigo relata os resultados obtidos com apoio de uma Avaliação Heurística de Usabilidade [Nielsen and Molich 1990], realizada em conjunto a uma medição de usabilidade, que contou com o uso da escala System Usability Scale (SUS) [Brooke 1996]. Ambas as atividades foram conduzidas com o intuito de identificar problemas de usabilidade no aplicativo, a partir da exploração de suas telas com o apoio de tarefas predefinidas. Tem-se como objetivo a retomada de seu desenvolvimento, aprimorando suas funcionalidades e interface de usuário, visando à evolução do software proposto.

Este artigo está organizado como segue. Na seção 2, apresentam-se conceitos teóricos e metodológicos para compreensão do trabalho. Na seção 3, delineia-se a metodologia utilizada para a condução das avaliações. Na seção 4, constam os resultados e as ameaças à validade que foram identificadas. Por fim, na seção 5, estão as considerações finais e os trabalhos futuros.

\section{Referencial Teórico-Metodológico}

O termo "usabilidade" é frequentemente usado quando se trata da capacidade que um produto tem de ser confortável e de fácil uso [Carroll 2013]. Essa ideia está diretamente relacionada aos princípios da Engenharia de Software, tendo em vista que todo produto de software desenvolvido deve suprir as necessidades e promover a satisfação de seus usuários, portanto possuir bons atributos de usabilidade [ISO/IEC 2001].

Para medir esses atributos e comprovar a qualidade de software, realizam-se atividades de verificação e validação. Estas podem ser conduzidas em qualquer momento do processo de desenvolvimento de software, por exemplo, pela avaliação de protótipos em diferentes níveis de fidelidade, desde maquetes até protótipos funcionais [Melo 2009]. Inspeções são um tipo de atividade de verificação e validação, possuindo grande importância, pois evitam que erros passem despercebidos, propagando-se durante o desenvolvimento e causando retrabalho para corrigi-los. Segundo [Felizardo 2004], a inspeção se mostra como um dos métodos mais efetivos para a garantia de qualidade de software.

Para avaliar a interface de usuário de um software, um dos métodos de inspeção conhecidos é a Avaliação Heurística de Usabilidade. Proposta por [Nielsen and Molich 1990], em 1990, consiste em um conjunto de dez heurísticas, apresentadas no Quadro 1, que devem ser analisadas durante o uso dessas interfaces. Essas avaliações são conduzidas com a participação de três a cinco especialistas e realizadas 
em duas fases. A primeira fase consiste em uma inspeção individual. Na segunda fase, os resultados obtidos na primeira são compilados, gerando uma única lista de problemas com seus respectivos graus de severidade. Essas duas fases permitem o levantamento de problemas decorrentes do uso e orientam suas respectivas correções.

\begin{tabular}{|l|l|c|l|}
\hline 1 & Visibilidade do estado do sistema. & 6 & Reconhecimento em vez de relembrar. \\
\hline 2 & Mapeamento entre o sistema e o mundo real. & 7 & Flexibilidade e eficiência de uso. \\
\hline 3 & Controle e liberdade do usuário. & 8 & Design estético e minimalista. \\
\hline 4 & Consistência e padrões. & 9 & Suporte para o usuário reconhecer, diagnosticar e recuperar erros. \\
\hline 5 & Prevenção de erros. & 10 & Ajuda e documentação. \\
\hline
\end{tabular}

Quadro 1. Heurísticas de usabilidade [Nielsen and Molich 1990]

A todos os problemas encontrados, devem ser atribuídos graus de severidade. Desse modo, os desenvolvedores podem priorizar a correção de erros no sistema em análise. Atualmente, são utilizados como parâmetro cinco graus de severidade, em uma escala de zero a quatro, apresentados no Quadro 2.

\begin{tabular}{|c|l|}
\hline 0 & Eu não concordo que isto seja um problema de usabilidade. \\
\hline 1 & $\begin{array}{l}\text { Problema apenas cosmético: não precisa ser corrigido a menos que haja tempo extra } \\
\text { disponível para o projeto. }\end{array}$ \\
\hline 2 & Problema de usabilidade menor: deveria ser dada baixa prioridade para sua correção. \\
\hline 3 & $\begin{array}{l}\text { Problema de usabilidade maior: importante corrigir e, assim, deveria ser dada alta } \\
\text { prioridade. }\end{array}$ \\
\hline 4 & Catástrofe de usabilidade: imperativo corrigi-la. \\
\hline
\end{tabular}

Quadro 2. Graus de severidade aplicados a problemas de usabilidade

Em sua obra, [Brooke 1996] propôs a escala SUS, que objetiva atribuir a um software, um grau de usabilidade. Essa escala auxilia na avaliação da efetividade, eficiência e satisfação do usuário ao utilizar interfaces. O uso da medida se dá a partir da resposta à dez questões, apresentadas no Quadro 3, respondidas com uso da escala Likert, de cinco pontos. Para atingir os resultados, há um calculo proposto por [Brooke 1996], feito em duas partes. Na primeira, deve-se subtrair 5 dos valores obtidos nas questões pares (2, 4, 6 e 8 ) e subtrair de 1 os valores obtidos nas questões ímpares (1,3. 7 e 9). Em seguida, os valores obtidos na primeira parte são multiplicados um a um por 2,5. Por fim, é calculada a média final, da qual obtemos a pontuação da SUS.

\begin{tabular}{|c|l|c|l|}
\hline 1 & $\begin{array}{l}\text { Eu acho que eu gostaria de utilizar este } \\
\text { sistema frequentemente. }\end{array}$ & 6 & Eu achei que tinha muito inconsistência no sistema. \\
\hline 2 & Eu achei o sistema desnecessariamente complexo. & 7 & $\begin{array}{l}\text { Eu acho que a maioria das pessoas iriam aprender } \\
\text { facilmente a utilizar esse sistema. }\end{array}$ \\
\hline 3 & Eu achei o sistema fácil de usar. & 8 & Eu achei o sistema muito incômodo de usar. \\
\hline 4 & $\begin{array}{l}\text { Eu acho que eu precisaria da ajuda de um técnico para } \\
\text { eu conseguir utilizar o sistema. }\end{array}$ & 9 & Eu me senti muito confiante em utilizar o sistema. \\
\hline 5 & $\begin{array}{l}\text { Eu acho que as várias funcionalidades do sistemas estão } \\
\text { bem integradas. }\end{array}$ & 10 & $\begin{array}{l}\text { Eu precisei aprender muito antes de utilizar } \\
\text { esse sistema. }\end{array}$ \\
\hline
\end{tabular}

\section{Quadro 3. Questões da escala SUS}

Segundo o autor, para que um aplicativo tenha uma usabilidade considerada boa, sua pontuação, obtida através da média final, deve estar acima de 68 pontos 
[Brooke 1996]. Para demonstrar os resultados da SUS de uma maneira diferente, [Sauro and Lewis 2016] propuseram uma escala alfabética, de acordo com as possibilidades de pontuação que um software pode obter. O Quadro 4 apresenta uma versão adaptada da proposta elaborada por [Sauro and Lewis 2016].

\begin{tabular}{|c|l|l|}
\hline Intervalo de Pontuação SUS & Grau & Percentual \\
\hline $84,1-100$ & $\mathrm{~A}+$ & $96-100$ \\
\hline $80,8-84,0$ & $\mathrm{~A}$ & $90-95$ \\
\hline $78,9-80,7$ & $\mathrm{~A}-$ & $85-89$ \\
\hline $77,2-78,8$ & $\mathrm{~B}+$ & $80-84$ \\
\hline $74,1-77,1$ & $\mathrm{~B}$ & $70-79$ \\
\hline $72,6-74,0$ & $\mathrm{~B}-$ & $65-69$ \\
\hline $71,1-72,5$ & $\mathrm{C}+$ & $60-64$ \\
\hline $65,0-71,0$ & $\mathrm{C}$ & $41-59$ \\
\hline $62,7-64,9$ & $\mathrm{C}-$ & $35-40$ \\
\hline $51,7-62,6$ & $\mathrm{D}$ & $15-34$ \\
\hline $0,0-51,6$ & $\mathrm{~F}$ & $0-14$ \\
\hline & &
\end{tabular}

Quadro 4. Interpretação da escala SUS

\section{Metodologia}

Com o intuito de inspecionar e avaliar a usabilidade do aplicativo ParecerEdu, foi planejado um experimento organizado em duas etapas. A primeira etapa consistiu em uma Avaliação Heurística de Usabilidade com o objetivo de identificar problemas de usabilidade a partir das heurísticas propostas por Nielsen [Nielsen and Molich 1990]. A segunda etapa, com foco em medição de usabilidade, contou com a aplicação do questionário de medida System Usability Scale, o qual todos os voluntários responderam. O uso dos dois métodos combinados permite a identificação e a classificação os problemas encontrados. Além disso, a atribuição da pontuação SUS permitirá mensurar a melhoria do app em trabalhos futuros.

Como critério de seleção dos voluntários, foi definido que estes deveriam ter cursado o componente curricular de graduação "Interação Humano-Computador", presente na matriz curricular do curso de Engenharia de Software do Campus Alegrete da Universidade Federal do Pampa (Unipampa). Os voluntários selecionados foram identificados em contato direto e pela demonstração de interesse. No total, foram selecionados três discentes do curso de Engenharia de Software (Unipampa), com os quais foram combinados dia e horário da condução do experimento. Para que os voluntários selecionados pudessem ter acesso ao aplicativo, este foi disponibilizado através do Google Drive junto a um manual de instalaçãa ${ }^{1}$.

O experimento foi executado no dia 21 de agosto de 2021, com início às $17 \mathrm{~h}$, com apoio de uma sala no Google Meet Institucional. Conduzido pela bolsista do GEIHC Grupo de Ensino em Interação Humano-Computador, do Programa de Desenvolvimento Acadêmico (PDA) da Universidade, contou com a participação dos voluntários selecionados. Com o intuito de evitar atrasos, os voluntários foram instruídos a realizar a instalação do aplicativo, seguindo os passos apresentados no manual, antes do início do experimento, com a condição de não usá-lo até começarem das atividades de avaliação.

\footnotetext{
${ }^{1}$ Acesso ao manual de instalação: https://bit.ly/3aLP4kg
} 
Para a execução da primeira etapa do experimento, foi elaborado um roteiro de tarefas ${ }^{2}$. Este, contém uma série de passos que deveriam ser seguidos pelos voluntários, visando cobrir todo o escopo de funcionalidades presentes no aplicativo. Nessa etapa, os voluntários foram instruídos a informar o horário de início e de fim da execução desses passos, para que fosse possível obter um prospecto referente ao tempo de execução das tarefas. Esse feedback é importante, pois com isso é possível compreender a complexidade de uso do aplicativo.

Para introduzir o experimento, foi realizada uma apresentação de slides ${ }^{3}$. Isso para que os voluntários relembrassem as Heurísticas de Usabilidade de Nielsen [Nielsen and Molich 1990] e pudessem, enquanto utilizavam o aplicativo, associá-las aos problemas identificados. Durante todo o processo, esses slides ficaram acessíveis aos voluntários.

Com o fim da apresentação, foi dado início à primeira etapa do experimento, com a execução dos roteiro de passos elaborado para a realização da avaliação. A cada um dos voluntários foi disponibilizada uma planilha no Google Drive, na qual eles deveriam anotar os problemas de usabilidade encontrados e a esses problemas atribuir um grau de severidade.

Após finalizado o roteiro de atividades, foi dado início à segunda parte do experimento, a qual consistiu no preenchimento de um formulário ${ }^{4}$ contendo as questões da escala SUS Quadro 3). Por fim, foi gerada uma lista com todos os problemas encontrados, onde a condutora e os voluntários avaliaram, um a um, individualmente atribuindo a graus de severidade.

\section{Resultados}

O primeiro item, analisado a partir dos resultados, foi o tempo médio dos voluntários para a realização das tarefas propostas. Com isso, constatou-se alta complexidade do sistema: para a realização de 11 tarefas, os voluntários demandaram, em média, 43minutos. Os tempos individuais de cada voluntário são apresentados na Tabela 1

Tabela 1. Tempo individual de execução da etapa 1

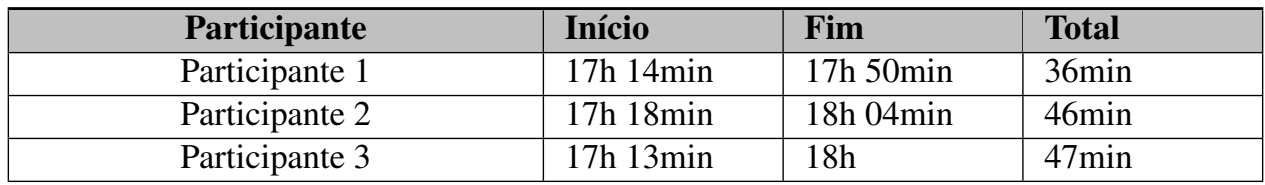

Da análise dos problemas reportados nas planilhas 5 , foi possível constatar quais heurísticas foram violadas de modo mais recorrente. Como pode ser observado no gráfico apresentado na Figura 1, a maioria dos problemas estão relacionados às heurísticas H4 Consistência e padrões, H7 - Flexibilidade e eficiência de uso e H8 - Design estético e minimalista.

No que se refere aos graus de severidade $e^{6}$ atribuídos aos problemas encontrados,

\footnotetext{
${ }^{2}$ Acesso ao roteiro de passos da avaliação: https://bit.ly/3aLP4kg

${ }^{3}$ Acesso aos slides apresentados: https://bit.ly/3aLP4kg

${ }^{4}$ Acesso ao formulário: https://bit.ly/3aLP4kg

${ }^{5}$ Acesso a lista de problemas encontrados: https://bit.ly/3aLP4kg

${ }^{6}$ Acesso aos graus de severidade atribuídos: https://bit.ly/3aLP4kg
} 


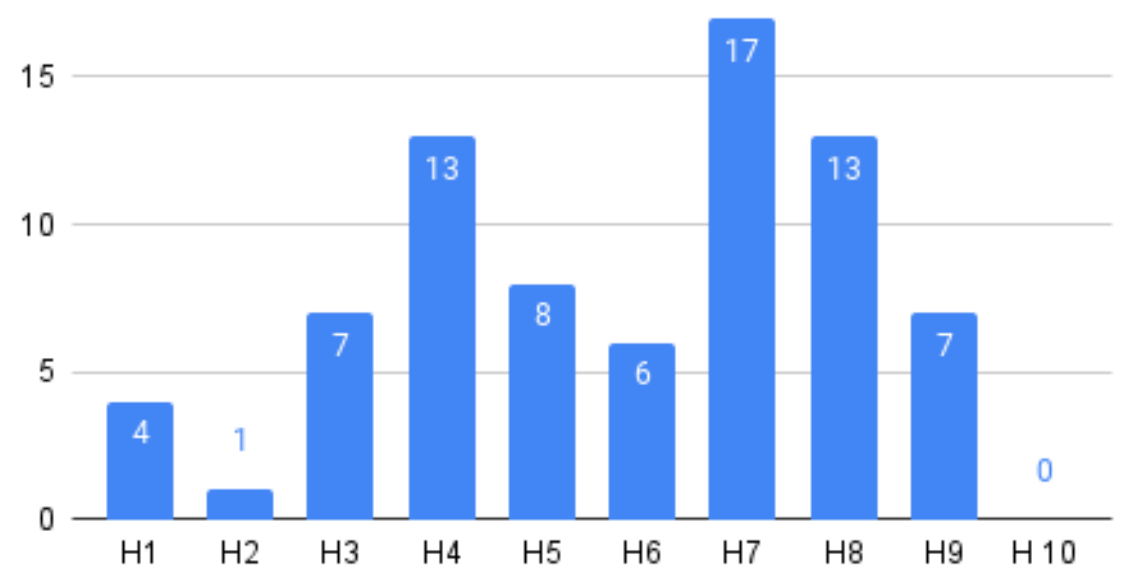

Figura 1. Número de problemas identificados por Heurística de Usabilidade

demonstrado na Figura 2, 42,9\% foram classificados com grau 3 e 27,7\% de grau 4 . Tendo isso em vista, pode-se dizer que a grande maioria dos problemas encontrados são graves.

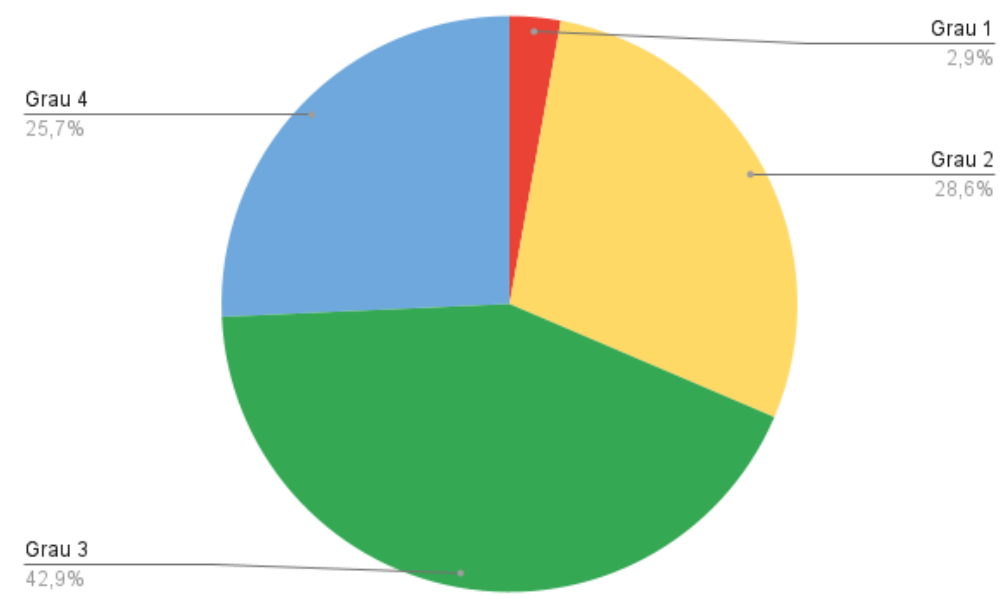

Figura 2. Distribuição dos problemas distribuídos por grau de severidade seguir.

Dos problemas identificados, os mais graves, com graus 3 e 4, são apresentados a Grau 3:

- P01 - Geral - O usuário do aplicativo não recebe nenhum tipo de feedback referente ao sucesso ou falha de suas ações no sistema;

- P02 - Tela de escolas - Após acessar uma escola, não é informado ao usuário em qual escola ele está;

- P03 - Gráficos - Não há limitação de casas decimais nos valores inseridos nos gráficos, dificultando a leitura do usuário;

- P04 - Exclusões e edições - Em nenhuma área do sistema ficam claras ao usuário as opções de edição e de exclusão de dados cadastrados; 
- P05 - Áreas do sistema - Nenhuma das páginas do sistema possui identificação e, assim, o usuário tem dificuldade em se localizar;

- P06 - Registros repetidos - Pode-se adicionar a mesma escola, turma, aluno, critérios e avaliação repetidas vezes.

Grau 4:

- P07 - Tela inicial - O usuário do aplicativo não possui cadastros. Sendo assim, suas informações e o trabalho realizado no aplicativo não têm persistência. Caso haja necessidade de troca de aparelho, todo o trabalho produzido no aplicativo é perdido;

- P08 - Tela de avaliação - Uma vez iniciada a avaliação de um critério, esta não pode ser parada com a opção cancelar. Caso isso seja feito, ao voltar para a mesma avaliação, o usuário não retorna ao estado onde havia parado, tendo que realizar a avaliação de todos os alunos novamente;

- P09 - Tela de avaliação - Um critério não pode ser avaliado novamente. Caso tenha sido cometido algum erro na avaliação, este deve ser recriado. Contudo, as opções de editá-lo ou excluí-lo não são claras ao usuário;

- P10 - Tela de alunos - O cadastro de um aluno, em uma turma, consiste apenas no registro de seu nome próprio. Com isso, em casos de nomes repetidos o professor deveria saber como distingui-los. As funções de exclusão e edição de alunos, apesar de existentes, não são claras ao usuário;

- P11 - Tela de escolas - Embora existam, não estão claras ao usuário as opções de edição ou de exclusão de escolas;

- P12 - Registros - Ao excluir um registro, em qualquer área do sistema, não é perguntado ao usuário se este tem certeza que deseja realizar a exclusão.

Para ilustrar alguns dos problemas mais graves citados, de grau 4, a Figura 3a, Figura 3b. Figura 4a e Figura 4b possuem pontos destacados e são relacionadas aos itens apresentados. Como pode ser observado, na Figura 3a, não há opções visíveis ao usuário no que se refere à edição ou à remoção de escolas (P05), sendo o único botão visível o de adição. Esta também se torna a tela inicial do aplicativo após o primeiro acesso. Já na Figura 3b, podemos observar os problemas mencionados no P04. Nessa tela temos como único campo para cadastro o nome do aluno.

Outro problema apresentado na tela de alunos (P04) é a ausência, visual ao usuário, de qualquer função de edição ou de exclusão de alunos (Figura 4a). Assim como na Figura 3a, a única opção visível é a adição de um novo aluno. Por fim, na Figura 4b, podemos observar que não há nenhuma função, explícita ao usuário, sobre edição ou exclusão do critério cadastrado.

A análise dos dados obtidos na segunda etapa se deu através do cálculo da medida SUS, já apresentado na Seção 3 . Após a realização dos procedimentos para a obtenção do grau de usabilidade do aplicativo ParecerEdu, constatou-se que este atingiu apenas 65,3 pontos. Segundo o autor da medida, com essa pontuação o cenário é de "problemas sérios de usabilidade no produto" [Brooke 1996]. Utilizando da interpretação proposta por [Sauro and Lewis 2016] (Quadro 4), o aplicativo atingiu grau C, estando com uma pontuação ruim, abaixo da média esperada. Sendo assim, há evidências suficientes que sustentam a necessidade de refatorações no aplicativo. 


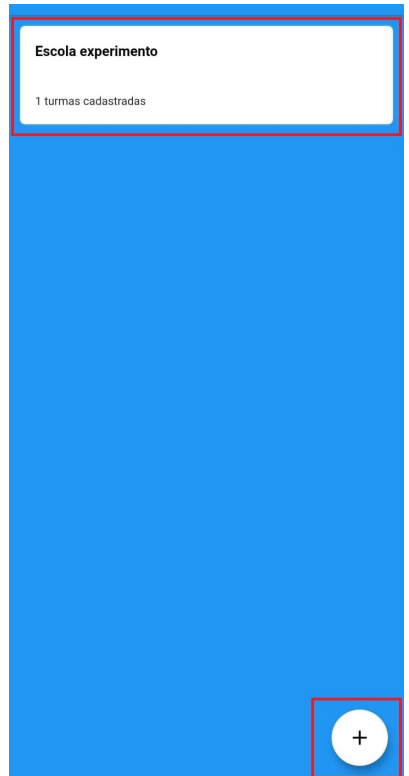

(a) Tela de escolas

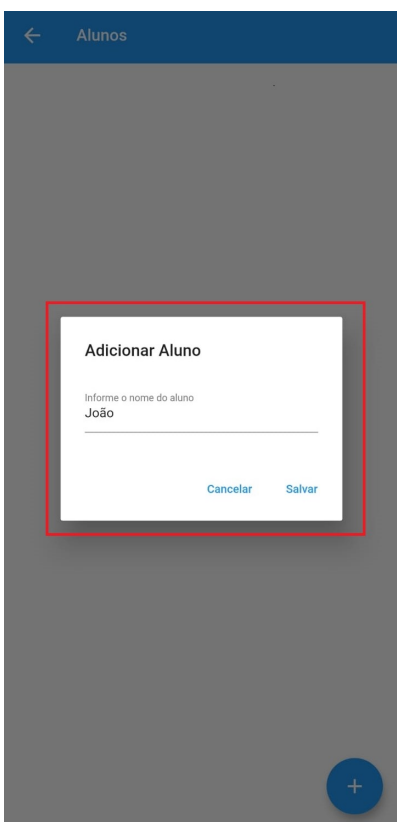

(b) Tela de adição de alunos

Figura 3. Telas de escola e cadastro de alunos - ParecerEdu.

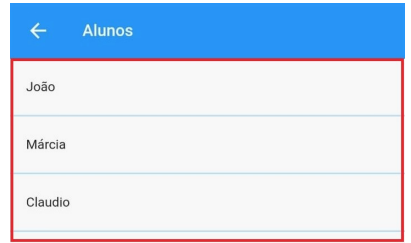

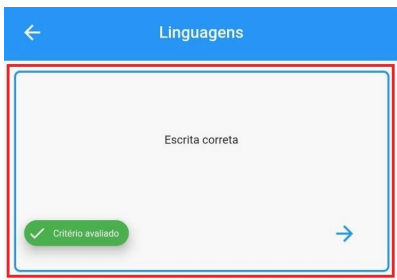

(a) Tela de alunos

(b) Tela de critérios

Figura 4. Telas de alunos e critérios - ParecerEdu. 


\subsection{Ameaças e Limitações}

Todo experimento possui ameaças e limitações que devem ser devidamente tratadas. Para contornar esse fato, deve-se encontrar medidas que possibilitem mitigar ou eliminar esses problemas.

A primeira ameaça identificada, para o presente estudo, foi a possível falta de conhecimento dos participantes, no que se refere às heurísticas propostas por Nielsen [Nielsen and Molich 1990]. Para mitigar essa ameaça, foi adotado um critério de seleção de voluntários, o qual incluía apenas alunos que já tivessem cursado o componente curricular de graduação "Interação Humano-Computador". Estes, por já terem aplicado o protocolo da Avaliação Heurística de Usabilidade, associariam mais facilmente os problemas identificados às heurísticas. Além disso, foi realizada uma apresentação das heurísticas, que também foi disponibilizada aos participantes. Assim, em casos de dúvidas, o material estava disponível para consulta.

Outra ameaça identificada foi o conhecimento dos participantes da aplicação em questão. A experiência de uso prejudicaria a identificação de problemas na ferramenta. Para eliminar essa ameaça, todos os participantes foram questionados quanto ao conhecimento prévio da aplicação e negaram já ter utilizado a ferramenta. Outra estratégia utilizada foi, ao disponibilizar o link para download, instruir os participantes a apenas utilizar a aplicação durante o período da avaliação.

Também foi identificado como ameaça o fato de o experimento ser realizado nos celulares pessoais dos voluntários. Esse fato poderia acarretar em discrepâncias no uso e flow da execução das atividades propostas. Para contornar a ameaça, os participantes puderam realizar o download da aplicação antes, garantindo que seus aparelhos suportavam o app.

Por fim, a última ameaça identificada foi o tamanho amostral de voluntários que participaram da avaliação. Avaliações heurísticas de usabilidade são conduzidas, geralmente, com o envolvimento de três a cinco participantes. No estudo em questão, participaram três voluntários. Essa decisão se deu pelo fato da obtenção de maior controle do experimento por parte da condutora. No que se refere ao uso da medida SUS, é importante reconhecer que ela reflete a avaliação do grupo envolvido no experimento e teve como referência o número de avaliadores recomendado para a Avaliação Heurística de Usabilidade.

\section{Considerações Finais}

Software de gestão podem ser de grande auxílio a profissionais da Educação, pois permitem maior controle dos dados gerados em sala de aula e entregam maior facilidade de manutenção desses dados. Pensando nisso, o aplicativo desenvolvido por [Oliveira and Melo 2020] é de grande relevância na área e possui muito potencial de uso. Contudo, toda ferramenta deve ser avaliada para que possa ser efetivamente adotada e evoluída.

Com a avaliação conduzida na ferramenta, puderam-se constatar diversos problemas de usabilidade. A maior parte deles com alto grau de severidade, indicando a necessidade de correções importantes. Sem que essas correções sejam feitas, o aplicativo não está apto a ser inserido no meio educacional, desperdiçando o trabalho produzido. 
Tendo isso em vista, o presente artigo visou demonstrar os resultados obtidos, para que seja possível trabalhar na evolução desse software.

Como trabalhos futuros, serão realizadas oficinas de prototipação, onde os problemas elicitados neste trabalho serão apresentados e correções serão propostas. Após essa etapa será possível dar início a refatoração do aplicativo. Deve-se pontuar que novas inspeções devem ser feitas ao longo do processo de evolução.

\section{Referências}

Brooke, J. (1996). SUS: a "quick and dirty" usability. Usability evaluation in industry, 189.

Carroll, J. M. (2013). Human computer interaction (hci). The Encyclopedia of HumanComputer Interaction, 2 nd Ed.

Felizardo, K. R. (2004). Apoio computacional para inspeção de software. INFOCOMP Journal of Computer Science, 3(2):14-18.

ISO/IEC (2001). ISO/IEC 9126. Software engineering - Product quality. ISO/IEC.

Melo, S. M. (2009). Inspeção de software. University of São Paulo: São Carlos, SP.

Muller, M. J., Haslwanter, J. H., and Dayton, T. (1997). Chapter 11 - participatory practices in the software lifecycle. In Helander, M. G., Landauer, T. K., and Prabhu, P. V., editors, Handbook of Human-Computer Interaction (Second Edition), pages 255-297. North-Holland, Amsterdam, second edition edition.

Nagar, M. A. K., Rahoo, L. A., Rehman, H. A., and Arshad, S. (2018). Education management information systems in the primary schools of sindh a case study of hyderabad division. In 2018 IEEE 5th International Conference on Engineering Technologies and Applied Sciences (ICETAS), pages 1-5.

Nielsen, J. and Molich, R. (1990). Heuristic evaluation of user interfaces. CHI '90, page 249-256, New York, NY, USA. Association for Computing Machinery.

Oliveira, I. and Melo, A. (2020). Pareceredu: um aplicativo para apoio à elaboração de pareceres nos anos iniciais de ensino fundamental. In Anais do XXXI Simpósio Brasileiro de Informática na Educação, pages 672-681, Porto Alegre, RS, Brasil. SBC.

Sauro, J. and Lewis, J. R. (2016). Chapter 8 - standardized usability questionnaires. In Sauro, J. and Lewis, J. R., editors, Quantifying the User Experience (Second Edition), pages 185-248. Morgan Kaufmann, Boston, second edition edition.

Sousa, R. (2011). Tecnologias digitais na educacao. EDUEPB, Campina Grande, Brazil. 